\title{
Hematoma de la vaina de los rectos abdominales
}

\author{
Juan Francisco Funes Rodríguez, ${ }^{1}$ José Camarena Martínez, ${ }^{2}$ Jorge Magaña Reyes, ${ }^{3}$ \\ Luis Gerardo Domínguez Carrillo ${ }^{4}$
}

\section{Resumen}

Antecedentes: El hematoma de la vaina de los músculos rectos abdominales es una causa poco frecuente de dolor abdominal; requiere de un diagnóstico acucioso que evita realizar laparotomía. Caso clínico: Paciente masculino de 40 años, quien al estar jugando balón-pie y realizar bruscamente hiperextensión tóraco-lumbar presentó de manera repentina dolor abdominal pungitivo en hipogastrio y tercio interno de ingle derecha, que le imposibilitó para continuar con su actividad. El dolor se incrementó requiriendo valoración por cirugía y encontrando: abdomen en madera, Blumberg positivo y análisis de laboratorio normales; el ultrasonido abdominal demostró hematoma en vaina de los rectos abdominales tipo II. Se manejó conservadoramente, requiriendo 60 días para poder efectuar ejercicios abdominales. Conclusiones: El hematoma de vaina de los rectos es un cuadro poco frecuente, pero debe ser parte del diagnóstico diferencial de dolor abdominal en los servicios de urgencias, pues su detección evita laparotomías innecesarias.

Palabras clave: Hematoma, músculos rectos abdominales.

${ }^{1}$ Cirujano General. División de Cirugía del Hospital Ángeles León. León, Gto., México.

2 Traumatólogo y Ortopedista. División de Cirugía del Hospital Ángeles León. León, Gto., México.

3 Radiólogo. Departamento de Imagenología del Hospital Ángeles León. León, Gto., México.

${ }^{4}$ Especialista en Medicina de Rehabilitación. Profesor de la Facultad de Medicina de León, Universidad de Guanajuato. México.

Correspondencia:

Dr. Luis Gerardo Domínguez Carrillo

Correo electrónico: Igdominguez@hotmail.com

Aceptado: 27-09-2016.

Este artículo puede ser consultado en versión completa en http:// www.medigraphic.com/actamedica

\section{Summary}

Background: Sheath hematoma of the rectus abdominis muscle is a rare cause of abdominal pain. It requires a careful diagnosis, which avoid laparotomy. Case report: A 40-year-oldmale playing football, performs an abruptly thoracolumbar hyperextension, presenting immediately a severe and sharp abdominal pain in lower abdomen and inner third of right groin, which made him impossible to continue the activity. Patient required surgery evaluation, finding: signs of local peritoneal irritation with rebound tenderness and involuntary guarding. Abdominal ultrasound showed hematoma in the rectus abdominis sheath type II. It was managed conservatively requiring 60 days to perform abdominal exercises. Conclusions: The hematoma rectus sheath, is an uncommon cause of abdominal pain, but should be part of the differential diagnosis of abdominal pain in emergency departments because diagnosis avoids unnecessary laparotomy.

Key words: Sheath hematoma, rectus abdominis muscles.

\section{INTRODUCCIÓN}

El hematoma de la vaina de los músculos rectos abdominales (HVR) es una causa poco frecuente de dolor abdominal que puede llegar a confundirse con abdomen agudo; es resultado de sangrado por lesión de los vasos epigástricos superiores o inferiores o de sus ramos perforantes debido a desgarro de los músculos rectos. Históricamente, el HVR fue descrito por Hipócrates y Galeno, y el primer reporte en la literatura anglosajona pertenece a Richardson ${ }^{1}$ en 1857; posterior a ello, existen algunas series de pacientes, siendo la mayoría reportes aislados, pero no escasos, ya que se encuentran 146 artículos relacionados con HVR en la literatura. Al presentarse un paciente en el que se llegó al diagnóstico de HVR realizamos el presente reporte.

\section{CASO CLÍNICO}

Paciente masculino de 40 años, sin antecedentes de importancia, quien inició padecimiento al estar jugando 
balón-pie en terreno lodoso, y que después de resbalar sufrió dolor abdominal pungitivo en hipogastrio y tercio interno de ingle izquierda, imposibilitándole para continuar con la actividad. Seis horas después, el dolor abdominal se incrementó, con irradiación urente a bolsa escrotal. Acudió a valoración por cirujano general, quien reportó: FC 72X', FR 18X', TA 125/80, peristalsis normal, hiperestesia en meso e hipogastrio, vientre en madera por espasmo de músculos abdominales, Blumberg positivo en meso e hipogastrio, tacto rectal con próstata normal, doloroso a la presión de pared anterior; no se encontró masa palpable; los análisis de laboratorio resultaron normales; se efectuó ultrasonido abdominal encontrando hematoma en vaina de los rectos abdominales de línea arcuata a $2 \mathrm{~cm}$ del pubis, con volumen calculado en $16.5 \mathrm{~cm}^{3}$ (Figuras 1 a 3), catalogado de tipo II; el estudio ecosonográfico de región inguinal y bolsa escrotal normal. Se solicitó interconsulta al Servicio de Rehabilitación, donde se prescribió: reposo, aplicación de hielo en meso e hipogastrio por lapsos de 20 minutos, con descansos de 30 minutos durante las primeras 24 horas, además de tramadol gotas $10 \mathrm{mg}$ qid, pregabalina $150 \mathrm{mg}$ sid, senocidos sid y dos litros de agua/día por vía oral, además de faja tóraco-pélvica con soporte abdominal amplio, de uso continuo, excepto para aplicación de hielo. Se realizó monitoreo del hematocrito cada ocho horas, que permaneció sin cambios. Al tercer día se permitió ambulación a tolerancia con órtesis colocada, indicando evitar maniobras de Valsalva; con nuevo ultrasonido de control, se corroboró no incremento del hematoma, dándose de alta de hospitalización; su evolución fue hacia la mejoría paulatina que llevó 60 días, para entonces poder efectuar ejercicios de contracción de músculos abdominales.

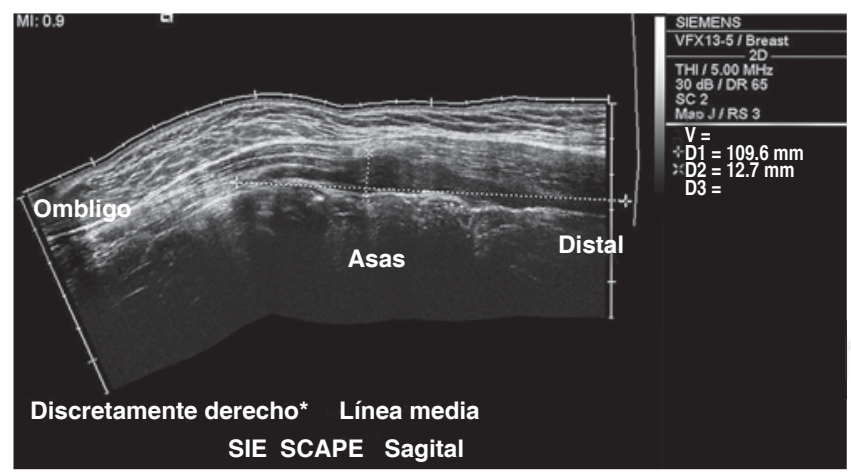

Figura 1. Imagen de ultrasonido en corte sagital que muestra hematoma en vaina de los rectos abdominales ocupando espacio entre la fascia transversalis y el peritoneo, con longitud $109.6 \mathrm{~mm}$ de la línea arcuata hasta $2 \mathrm{~cm}$ por arriba del pubis, con dimensión antero-posterior de $12.7 \mathrm{~mm}$.

\section{DISCUSIÓN}

La irrigación de los músculos rectos abdominales es proporcionada por las arterias epigástricas, ${ }^{2}$ la superior (rama de la arteria torácica externa) por arriba de la línea arcuata, y la epigástrica inferior (rama de la arteria iliaca externa) por debajo de ella, ambas arribando al músculo por su cara posterior; la inervación es proporcionada de manera segmentaria por los ramos intercostales y abdominales; en la parte inferior arriban las ramas terminales de los nervios abdominogenitales superior e inferior (en el caso

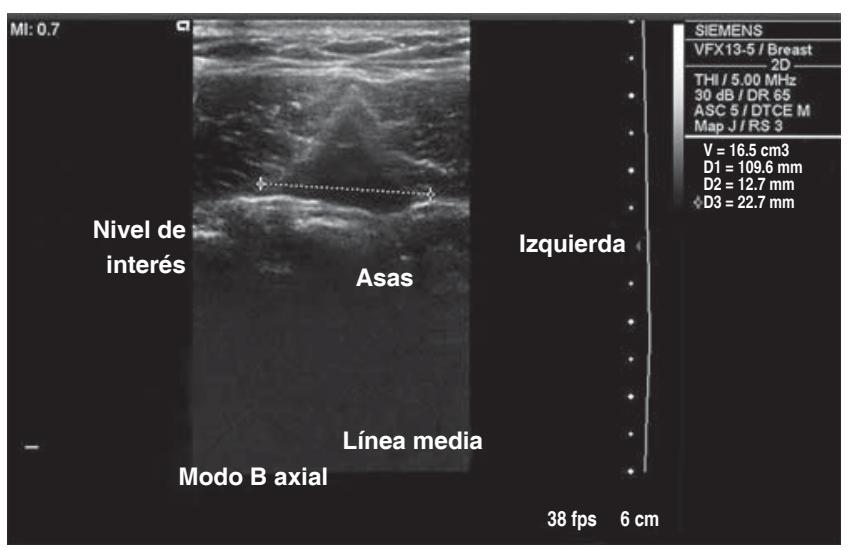

Figura 2. Imagen de ultrasonido de pared abdominal, a nivel de espinas iliacas anterosuperiores, en corte axial, donde se muestra hematoma de forma romboidal en línea media con dimensiones de $22.7 \mathrm{~mm}$, y volumen calculado en $16.5 \mathrm{~cm}^{3}$, con ruptura de la fascia transversalis del músculo recto anterior izquierdo y desgarro del mismo.

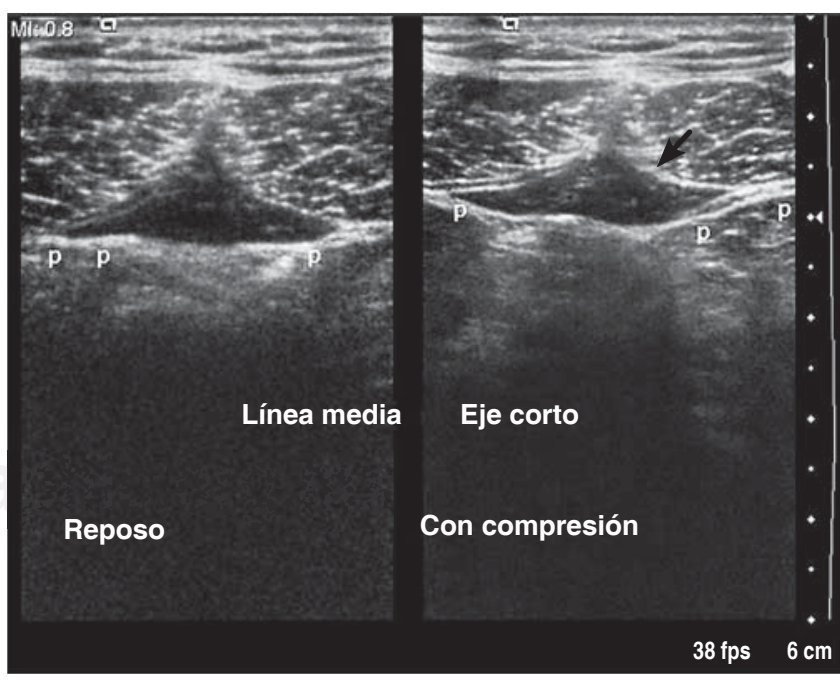

Figura 3. Imagen de ultrasonido de pared abdominal en corte axial, en reposo y con compresión, donde se observa ruptura de la fascia transversalis en músculo recto anterior izquierdo. 
presentado, la irritación de dichos nervios originó el dolor urente en tercio interno de la ingle y de la bolsa escrotal).

Fisiopatológicamente, el HVR es originado por lesión de alguna de las arterias mencionadas o de sus ramos perforantes, durante el desgarro de los rectos abdominales. La mayoría de los HVR se presentan en la cara posterior de los rectos, haciendo más difícil su diagnóstico por palpación. Por arriba de la línea arcuata, el HVR queda confinado por las fuertes aponeurosis de los músculos, que además auxilian al taponamiento del sangrado por inextensibilidad, llegando a causar síndrome compartimental de los rectos, mientras que por debajo de ella el hematoma puede ser de mayor cuantía irritando al peritoneo (confundiendo con abdomen agudo) y disecando el espacio de Retzius.

La incidencia del HVR es de $1.8 \%$, incrementada en pacientes con uso de anticoagulantes; se le ha reportado en edades entre cuatro y 83 años, siendo más frecuente a los 47 años, prevaleciendo en el género femenino con relación 2-3:1, siendo menos frecuente en afroamericanos. ${ }^{3}$

Los factores de riesgo involucrados ${ }^{4}$ en el HVR incluyen: uso de anticoagulantes, trauma directo de abdomen, discrasias sanguíneas, accesos de tos intensa, enfermedad renal crónica, halterofilia, iatrogenia durante cirugía laparoscópica, inyecciones en abdomen; en 2007 Maquirriain ${ }^{5}$ reportó el HVR en jugadores profesionales de tenis, ocasionado durante el movimiento de envío del servicio por el estiramiento y posterior contracción intensa de los abdominales (situación semejante a la que se presentó en el paciente motivo de este reporte); el HVR puede asociarse al embarazo, durante el parto y en periodo postparto temprano.

Las manifestaciones clínicas del HVR varían, desde dolor moderado localizado en la pared abdominal anterior al realizar maniobra de Valsalva, hasta dolor intenso de tipo pungitivo (80 a 97\% de los casos), acompañado de náuseas (23\%), vómito (15\%) y fiebre; en la exploración se puede detectar masa abdominal en 63 a $90 \%$ de los pacientes, hiperestesia de pared abdominal en $71 \%$ y signos de defensa abdominal en $49 \%{ }^{6}$ (como en este paciente).

Cuando existe HVR de gran magnitud, la pérdida de sangre puede causar síntomas de hipovolemia; ocasionalmente puede presentarse obstrucción urinaria e irritabilidad vesical; rara vez se observa equimosis de la pared abdominal; los signos de Cullen y Grey Turner pueden ser encontrados, pero cuando se presentan habitualmente son tardíos. ${ }^{7}$

El diagnóstico diferencial debe efectuarse con: abdomen agudo, apendicitis, colecistitis, diverticulitis, hernia estrangulada, hernia de Spiegel, obstrucción intestinal, vólvulos, aneurisma y tumor abdominal.

Los exámenes de laboratorio habitualmente son normales; la valoración seriada del hematocrito permite valorar si el sangrado continúa; las pruebas de coagulación deben ser efectuadas en todos los pacientes.
El ultrasonido de abdomen proporciona un abordaje inicial en el diagnóstico de dolor abdominal; en el caso de HVR permite, además de hacer diagnóstico (con sensibilidad de 85 a $96 \%$ ), monitorizar el hematoma. ${ }^{8}$

La tomografía de abdomen en el HVR tiene sensibilidad y especificidad del $100 \%$, mostrando una masa hiperdensa, localizada en los rectos abdominales; cuando el hematoma es crónico, la masa puede aparecer isodensa o hipodensa al compararla con el tejido adyacente. La clasificación tomográfica de Berna ${ }^{9}$ del HVR consta de tres tipos: en el tipo I, el hematoma es intramuscular y unilateral, sin disecar los planos fasciales y sin compromiso hemodinámico; el tipo II se caracteriza por hematoma intramuscular con sangre localizada entre el recto abdominal y la fascia transversalis (puede presentarse disminución del hematocrito); el tipo III es severo, con sangre que se extiende a la superficie del peritoneo y al espacio pre-vesical. Es importante recordar que la resonancia magnética en el caso de HVR no muestra señal de alta intensidad, por lo que en las primeras 48 horas no es de utilidad diagnóstica. ${ }^{10}$

En relación al tratamiento del HVR, ${ }^{11}$ depende de los datos clínicos y del estado hemodinámico del paciente; los pacientes con uso de anticoagulantes deben hospitalizarse y asegurarse que el hematoma no se esté expandiendo; los HVR de tipo I no requieren de hospitalización; los de tipo II deben vigilarse en hospitalización por 48 horas con monitoreo del hematocrito y ultrasonido; los de tipo III, casi siempre presentan inestabilidad hemodinámica, requiriendo resucitación y transfusión sanguínea. ${ }^{12}$ La gran mayoría de los pacientes pueden ser manejados con tratamiento conservador, incluyendo reposo y analgésicos, aplicación de crioterapia y evitar maniobras de Valsalva. ${ }^{13}$ En los casos en que se requiere tratamiento quirúrgico, éste implica: ligadura del vaso sangrante, reparación de la vaina de los rectos y drenaje cuando esté indicado; en general no se reportan recidivas. Actualmente se puede recurrir a la embolización del vaso sangrante por el radiólogo intervencionista. Se menciona la utilidad de aspiración del hematoma guiada por ultrasonido, existiendo el riesgo de contaminación bacteriana. ${ }^{14}$

En general, el pronóstico es bueno; los HVR de tipo I se resuelven en un mes, los de tipo II requieren de dos a cuatro meses (el paciente necesitó 60 días); los de tipo III pueden requerir de cirugía para su resolución incluyendo al menos tres meses para su total resolución; en este último grupo se reporta mortalidad de $4 \%$ y en caso de anticoagulación y necesidad de cirugía ésta se puede incrementar al 18\%.

\section{CONCLUSIONES}

El hematoma de vaina de los rectos es un cuadro poco frecuente, pero debe ser parte del diagnóstico diferencial 
de dolor abdominal en los servicios de urgencias, pues su diagnóstico evita laparotomías innecesarias.

\section{REFERENCIAS}

1. Richardson SB. Rupture of the right rectus abdominis muscle from muscular efforts: operation and recovery, with remarks. Am J Med Sci. 1857; 33: 41-45.

2. Jones TW, Merendino KA. The deep epigastric artery: rectus muscle syndrome. Am J Surg. 1962; 103: 159-169.

3. Cherry WB, Mueller PS. Rectus sheath hematoma: review of 126 cases at a single institution. Medicine (Baltimore). 2006; 85 (2): 105-110.

4. Sheth HS, Kumar R, DiNella J, Janov C, Kaldas H, Smith RE. Evaluation of risk factors for rectus sheath hematoma. Clin Appl Thromb Hemost. 2016; 22 (3): 292-296.

5. Maquirriain J, Ghisi JP, Kokalj AM. Rectus abdominis muscle strains in tennis players. Br J Sports Med. 2007; 41 (11): 842-848.

6. Hatjipetrou A, Anyfantakis D, Kastanakis M. Rectus sheath hematoma: a review of the literature. Int J Surg. 2015; 13: 267-271.

7. Toyonaga J, Tsuruya K, Masutani K, Maeda H, Nakamura K, Taniguchi $M$ et al. Hemorrhagic shock and obstructive uropathy due to a large rectus sheath hematoma in a patient on anticoagulant therapy. Intern Med. 2009; 48 (24): 2119-2122.

8. Lewiss RE, Wu S. Ultrasound diagnosis of rectus sheath hematoma. J Emerg Med. 2012; 43 (5): e337-e338.

9. Berná JD, Garcia-Medina V, Guirao J, Garcia-Medina J. Rectus sheath hematoma: diagnostic classification by CT. Abdom Imaging. 1996; 21 (1): 62-64.

10. Stoker J, van Randen A, Laméris W, Boermeester MA. Imaging patients with acute abdominal pain. Radiology. 2009; 253 (1): 31-46.

11. Buffone A, Basile G, Costanzo M, Veroux M, Terranova L, Basile A et al. Management of patients with rectus sheath hematoma: personal experience. J Formos Med Assoc. 2015; 114 (7): 647-651.

12. Zehtabchi S, Sinert R, Goldman M, Kapitanyan R, Ballas J. Diagnostic performance of serial haematocrit measurements in identifying major injury in adult trauma patients. Injury. 2006; 37 (1): 46-52.

13. Berná JD, Zuazu I, Madrigal M, García-Medina V, Fernández C, Guirado F. Conservative treatment of large rectus sheath hematoma in patients undergoing anticoagulant therapy. Abdom Imaging. 2000; 25 (3): 230-234.

14. Anyfantakis D, Kastanakis M, Petrakis G, Bobolakis E. Rectus sheath hematoma in a single secondary care institution: a retrospective study. Hernia. 2015; 19: 509-512. 